\title{
Machine Learning-Based Secure Data Acquisition for Fake Accounts Detection in Future Mobile Communication Networks
}

\author{
B. Prabhu Kavin, ${ }^{1}$ Sagar Karki, ${ }^{2}$ S. Hemalatha, ${ }^{3}$ Deepmala Singh, ${ }^{2}$ R. Vijayalakshmi, ${ }^{4}$ \\ M. Thangamani, ${ }^{5}$ Sulaima Lebbe Abdul Haleem, ${ }^{6}$ Deepa Jose, ${ }^{7}$ Vineet Tirth, ${ }^{8}$ \\ Pravin R. Kshirsagar $\mathbb{D},{ }^{9}$ and Amsalu Gosu Adigo $\mathbb{D}^{10}$
}

${ }^{1}$ Sri Ramachandra Institute of Higher Education and Research and Technology, Chennai, India

${ }^{2}$ LBEF Campus (In Academic Collaboration with APU Malaysia), Kathmandu, Nepal

${ }^{3}$ Department of Computer Science and Engineering, Panimalar Institute of Technology, Chennai, India

${ }^{4}$ Department of Computer Science and Engineering, Velammal College of Engineering and Technology, Madurai, India

${ }^{5}$ Department of Information Technology, Kongu Engineering College, Perundurai, Tamil Nadu, India

${ }^{6}$ Department of Information \& Communication Technology, South Eastern University of Sri Lanka (SEUSL), Sri Lanka

${ }^{7}$ KCG College of Technology, Karapakkam, Chennai, Tamil Nadu, India

${ }^{8}$ Mechanical Engineering Department, College of Engineering, King Khalid University, 61411 Abha, Asir, Saudi Arabia

${ }^{9}$ Department of Artificial Intelligence, G. H Raisoni College of Engineering, Nagpur, India

${ }^{10}$ Center of Excellence for Bioprocess and Biotechnology, Department of Chemical Engineering, College of Biological and Chemical Engineering, Addis Ababa Science and Technology University, Ethiopia

Correspondence should be addressed to Pravin R. Kshirsagar; pravinrkshirsagarphd@gmail.com and Amsalu Gosu Adigo; amsalu.gosu@aastu.edu.et

Received 20 December 2021; Revised 25 December 2021; Accepted 29 December 2021; Published 27 January 2022

Academic Editor: Deepak Kumar Jain

Copyright (c) 2022 B. Prabhu Kavin et al. This is an open access article distributed under the Creative Commons Attribution License, which permits unrestricted use, distribution, and reproduction in any medium, provided the original work is properly cited.

\begin{abstract}
Social media websites are becoming more prevalent on the Internet. Sites, such as Twitter, Facebook, and Instagram, spend significantly more of their time on users online. People in social media share thoughts, views, and facts and create new acquaintances. Social media sites supply users with a great deal of useful information. This enormous quantity of social media information invites hackers to abuse data. These hackers establish fraudulent profiles for actual people and distribute useless material. The material on spam might include commercials and harmful URLs that disrupt natural users. This spam content is a massive problem in social networks. Spam identification is a vital procedure on social media networking platforms. In this paper, we have proposed a spam detection artificial intelligence technique for Twitter social networks. In this approach, we employed a vector support machine, a neural artificial network, and a random forest technique to build a model. The results indicate that, compared with RF and ANN algorithms, the suggested support vector machine algorithm has the greatest precision, recall, and Fmeasure. The findings of this paper would be useful in monitoring and tracking social media shared photos for the identification of inappropriate content and forged images and to safeguard social media from digital threats and attacks.
\end{abstract}

\section{Introduction}

In the last few years, online social networks (OSNs), including Facebook, Twitter, and LinkedIn, are becoming extremely common. People use OSNs to remain in contact, exchange details, plan activities, and even operate their e-business [1].
The data set created has been preprocessed to identify false accounts on social networking sites, and the intelligent systems have identified false accounts. Random forest, neural network, and help vector machine classification output is used to identify fraudulent accounts. The precision rates of fake accounts are compared using certain algorithms, and the 
method is indicated with the highest accuracy [2]. In the past twenty years, social media have expanded exponentially. Various forms of social networking gained a vast amount of people, several events have been created, and social networking has a tone of misleading profiles and bogus news created. Also, the false accounts use their accounts for multiple aims, including circulating rumors that impact a certain economy or even culture as a wider market. The identification of news of deception is an ongoing problem [3]. Twitter is a large type of online communication that probably contains vast knowledge which opens up new opportunities for tweet content analysis. In reality, 74 per majority of people state that either the "lacking of IT infrastructure" or an overarching cost-benefit study is the main barrier to use technology. Despite these obstacles, technology appears to be gradually being embraced. More than half of the insurers analyzed said in the last five years, several in the last two years, they have been utilizing antifraud technology solutions $[2,3]$.

Twitter has many options to submit spam to address assaults by hackers. By clicking on the link, a web user can detect spam on their webpage. Twitter will evaluate the network user reports and deactivate the spam profiles. The Twitter network is working to reveal fraudulent messages and suspect reports efficiently [4]. Several real login credentials are blocked out by Twitter when you block harmful tweets and suspect profiles. We thus need to get some effective ways for trash and spammers to be detected instantly. These modern techniques have in meantime no impact on authentic user tweets. We have suggested in this paper an approach to detecting fraudulent social accounts. We utilized the Twitter data set in this paper [5]. The data set obtained is utilized to produce a normal data collection. Content-based features and user-based features were the types of features that were retrieved. To develop a model with these features, we are employing a support vector machine, an artificial neural network, and the random forest algorithm $[1,6]$.

\section{Objective}

In today's modern social networks, there have been numerous issues such as fraudulent profiles, online impersonation, and other similar issues. In this paper, I plan to highlight a conceptual model for the automatic recognition of fake accounts, to ensure that person's online lives are protected. We can also make it much simpler for sites to manage a larger amount of accounts by utilizing artificial intelligence techniques, which is incredibly difficult to accomplish manually at the moment due to a lack of resources.

\section{Threats}

As an online social network (OSN) is widely used, many consumers are vulnerable to both privacy and protection risks unequivocally. These risks may be grouped into four primary groups [7].

(i) The first group involves classic risks to privacy and protection, which not only target OSN members but even web users who do not use social networking sites $[7,8]$

(ii) The second column describes emerging risks, including attacks that are essentially new to the OSN ecosystem and use OSN technology to threaten the security and anonymity of users

(iii) The third group is combined risks, explaining how hackers today can, and sometimes do, merge multiple styles of threats to produce complex and deadly attacks [4]

(iv) The fourth and last types contain risks against children directly using social networks

Figure 1 illustrates all the risks in the parts below. However, the limitations between several risks may be obscured as strategies and goals sometimes overlap.

3.1. Classic Threats. Since the popularity of the Internet, classic attacks have become a concern. They appear to be a persistent problem also named ransomware assaults, spam, cross-site (XSS), and phishing. While these challenges have been discussed previously, depending on the structure and existence of OSNs, such threats have become highly viral and may easily propagate to network users [8]. Classic threats may manipulate the personal details of the user-posted in a social network not just to target the user but also his friends simply by modifying the threat to credit guarantee details of the user [5].

(i) Malware. Malware is software intended to disturb a device process to capture user passwords and gain entry to your privacy. Social network malware uses the OSN framework to spread across members and their network mates. In certain instances, the ransomware may use the passwords acquired to imitate the client and deliver emails to online contacts [9]

(ii) Phishing Attacks. A phishing attack is a type of social engineering, which allows a reputable third party to obtain user-sensitive and personal details. New research has found people who are more prone to be phishing scams because of their social and trustworthy nature, engaging with social media platforms. One assault was perpetrated on Facebook and drew people to the bogus Facebook login sites. The assault then spread among Facebook users by encouraging buddies to click on the link on the initial user profile [7]. Luckily, this assault was prevented by Facebook

(iii) Cross-Site Scripting (XSS). An intrusion from the XSS is a web-based assault. The intruder who uses XSS abuses the website client's confidence and lets the client's computer run spyware to gather confidential details

3.2. Modern Threats. These risks are typically linked with social networking online. In contrast to your connections, you want to collect users' details. Attackers on social networking sites like Twitter aim at the confidentiality of a user 


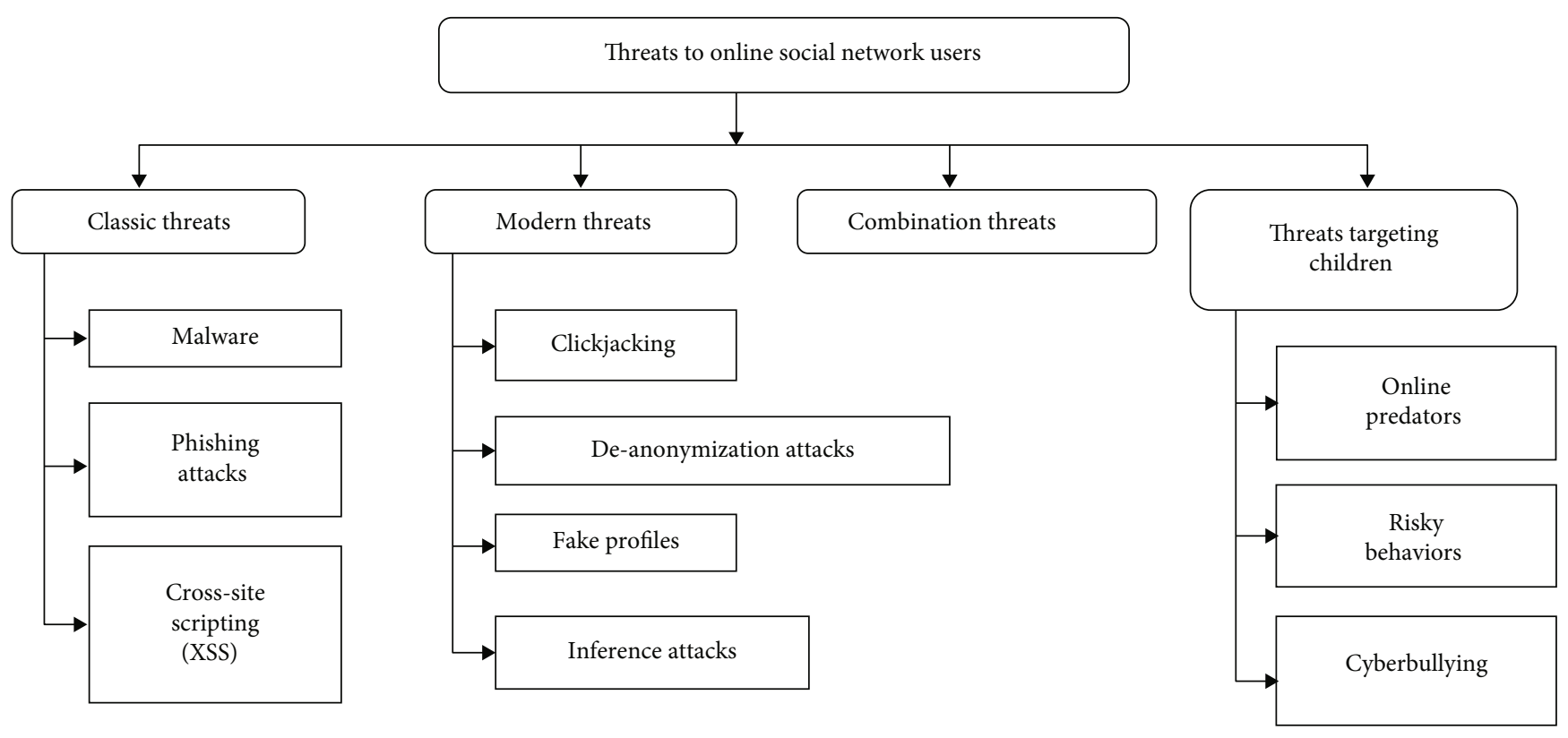

FIGURE 1: Threats to online social network users.

since this is highly essential for them. This allows an attacker to access this data anymore if confidential information is made public $[2,3]$; otherwise, an attacker can send a friend's request to certain users who have a tailored configuration. After then, your confidential information will be communicated on confirmation of the request for friendship by the specific attack. Below are the most advanced threats.

(i) Clickjacking. Clickjacking is a malware process that tricks users to click on something else click on. By pressing revving, the intruder will use spam notifications on its Facebook wall to exploit the client and unintentionally execute "like" connections [9]

(ii) Deanonymization Attacks. Users will secure their privacy and confidentiality using pseudonyms in certain OSNs such as Twitter and MySpace. Deanonymization attacks use cookie monitoring, network architecture, and user community affiliation methods to expose the true identity of the user $[7,8]$

(iii) Fake Profiles. Active or semiautomatic profiles (also known as styles or social bots) simulate human actions in OSNs. Fake accounts may also be used to gather personal details from social networking sites from the members. When you start connection requests for other people in the OSN, who also grant requests, social bots may be able to capture private user details that should be only accessible to friends of the user [4]

(iv) Inference Attacks. Inference attacks are used in OSNs to forecast users' confidential, private details, such as religious affiliation or sexual identity that they did not want to reveal. Such attacks can be carried out using data mining methods in conjunction with accessible public OSN data, such as the entire network and user friends' data [7]

3.3. Combination Threats. To build a more complex threat, today the attackers may still mix classical and contemporary menaces. For example, a phishing attack can be used by an intruder to capture a Facebook user password, then post messages with a clickjack on the stated schedule, so that friends of the user Facebook can click on a posted message and get a secret virus installed according to their own devices [10]. An additional example is the usage of cloned accounts to obtain personal details on cloned user mates. The attacker could submit special, personalized spam emails containing a virus using confidential info given by his friends. The malware is much more likely to be triggered when utilizing personal details [8].

3.4. Threats Targeting Children. Children, small children or teens, definitely encounter the above specifics of classical and contemporary threats, but some threats target younger OSN users deliberately and in particular $[4,10]$.

(i) Online Predators. The biggest issue about the privacy of children's confidential details is the Internet child predators, commonly known as cyber predators. To better understand the danger and harm associated with the next online events, EU Kids Online's Livingstone and Haddon described typology [4]

(ii) Risky Behaviors. Children's possible dangerous habits can involve overt Internet contact with foreigners, the usage of discussion forums for foreigner encounters, sexually provocative conversations with foreigners, and providing private details and images to foreigners

(iii) Cyberbullying. Cyberbullying (also known as cyber abuse) is an intruder that uses the web to annoy the 
victim by sending hurtful texts, lewd comments or intimidating multiple occasions, posting embarrassing images or videos of the victim, or participating in other offensive behaviors inside a technology network such as e-mail, talk, mobile conversations, and OSNs $[1,6]$

\section{Literature Review}

To provide effective identification on bogus Twitter account and bots, function selection technologies, and dimensional reduction strategies, [1] implemented a modern SVM-NN algorithm. This suggested methodology (SVMNN) uses fewer than $98 \%$ of the records of our training set but is still able to properly classify.

Regarding fake accounts on social media, particularly on Facebook, the technology of 2018. A computer training function was used in this study to help predict counterfeit accounts from their comments and the location on their walls. Suitable for validating material based on classification and interpretation of the text was used to support vector machine (SVM) and supplement naïve bays (NCB). In [3] suggested spacetime mining in the social grids, with latent semantical analytics, to classify the circle of consumers interested in malicious incidents. Then, compare the effects of spatial-temporal coincidence with the results of the initial organization/stories on the social network, as the wavelet covalue and real organization will produce very motivating covalue.

A proof of concept enhancer model was developed [6] which is successfully used for the identification of bots. In [9] detected spam in SMPs and used the value of features in iterating a higher output collection of laws. Machine learning methods require environmental input to be adapted and improved. In [7] effectively training a neural network in the analysis of the error level of 4000 false and 4000 actual images. With a strong success rate, the qualified neural network has managed to classify the picture as false or true.

A review of hackers on Twitter was proposed [8] to help grasp their behavioral features. An one hundred - thousand messages have been received to carry out the analysis over one month. The assessment was made of two separate spammer types using different trolling techniques. Also, three key groups identified a series of tools for identifying spammers: profile characteristics, social connections, and account assets. In [4], Facebook users have shown themselves to consider friendship invites from strangers they may not know but with several relatives. Users inadvertently divulge their private knowledge to absolute foreigners by taking in these demands from friends.

In $[6,11,12]$ elaborate on the use of artificial intelligence for different classification and prediction problems and furthermore explain the use of hybrid artificial intelligence for feature extraction, classification, and prediction along with modeling with different algorithms and optimization techniques [13].

In [10], he addressed the interest of making progress in the effective recognition of false identities produced by people on SMPs and applied them to a series of fake human accounts.

\section{Proposed System for Detecting Fake- Accounts in Twitter Using AI}

We utilized many approaches for spam detection in Twitter data in the proposed method as shown in Figure 2. Each approach employs its data set and data categorization functionality. Spam detection methods made use of a variety of forms of functionality, including user-based and contentbased features and graphs, among others. The advantages and disadvantages of each extracted feature are addressed $[10,11]$. We use these characteristics to develop a classification method that distinguishes between false information and information that is true. To get the best classification results, we created an integrated classification model that includes support vector machines, artificial neural networks, and the random forest approach.

5.1. Data Collection. There are two ways to gather the data set needed for experimental evaluation. The first step was to manually collect the information. Here, users collect the information that is present and designate them manually. A Twitter account with 1150 followers is utilized to gather the data manually. These were the real accounts [12]. User profile data is collected via the Twitter REST API. Sets of three persons perform additional labeling and verification. Another data set of the project "The Fake Project" was obtained, and it was incorporated together with the data obtained. The final data set consists of 7,973 account information, divided into two portions, $75 \%$ used for model training and 25\% used for model testing [14].

5.2. Data Preprocessing. The obtained information must be preprocessed through multiple measures until entering every classifier to ensure the algorithm recognizes the data and creates the absolute best model. Formatting and data cleaning are one of the preprocessing activities [11]. Formatting is an essential method by which the data can be read acceptably for the classifier, for example, by translating the data type into a text file or a flat format. Cleaning method managing the missing values of a data set, like missing labels or values of certain data, set properties that are manually accomplished by plurality voting for the matching values of other instances and even by deleting certain instances that adversely influence the classifier learning process [12]. Furthermore, cleaning details means deleting personal details that may breach the privacy of some people.

5.2.1. Tokenization. Tokenization is the breakdown of a textbased circulation into words, sentences, symbols, or various essential components known as tokens. The objective is to explore sentences in one phrase. The token list becomes a parsing input or a text-based mining input for further analysis. In languages (where textual material is segmented in a format) and in laptop technology, tokenization is valuable as a component of reading passages $[10,11]$. Textual knowledge at the beginning is most basic. All recognized recovery techniques need data set terms. For this purpose, a processor has to tokenize the data. This might be easy since the text is already recorded in readable codecs in the computer system. 


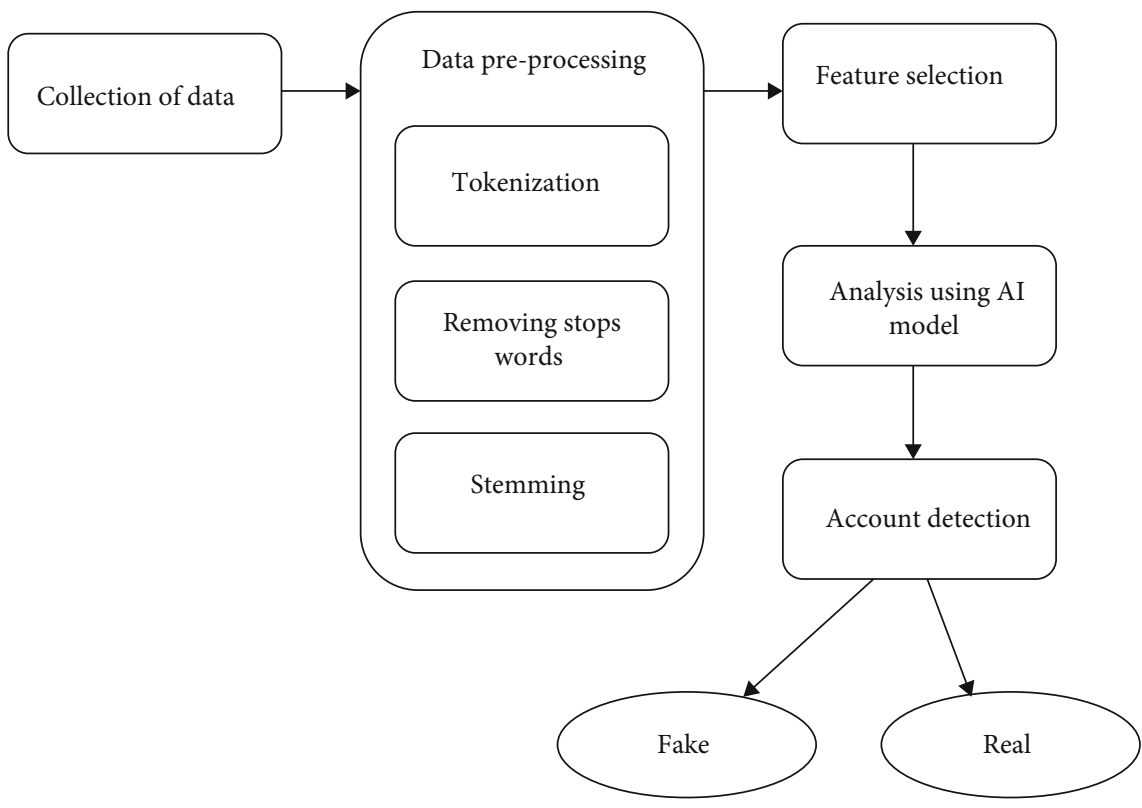

FIGURE 2: Fake account detection in Twitter using artificial intelligence.

Nevertheless, certain issues remain, such as deleting punctuation marks. Various characters such as brackets, hyphens, and others also have to be processed [12].

5.2.2. Stop Word Removal. Stop words are more common than conventional phrases like "and," "are," etc. They do not appear useful for the basis of the data collected. They must thus be eliminated. Moreover, the evolution of such stopping words between both text documents is complicated and uneven [15]. This method minimizes text knowledge and enhances the performance of the approach. Each textual content report includes these sentences that are not essential for solutions for textual data.

5.2.3. Stemming. Stemming is a primitive intuitive procedure that cuts off the extremities of words to attain this aim more often than not properly [7]. It frequently includes the removal of prefixes and suffixes, which is a common occurrence in the English language.

5.3. Feature Selection. Eleven characteristics have been discovered in the proposed spam detection approach. The retrieved characteristics are split into two categories.

5.3.1. User-Based Features. The activity of Twitter users is characterized using user-based characteristics, which are attributes that are unique to each user. These characteristics are based on data from the Twitter data set, which includes user relationships and user profiles, among other things. It is usual for users to collaborate with some other users on online communities to build their social networking sites. Phishers would like to follow numerous accounts [13]; thus, they try to track numerous people to spread the disinformation. They wish to track the fraudsters. Usually, we assume that the number of people that follow him is greater than that of users who follow him. To construct a model, we make use of several user-based features [16]. User functionality is associated with user profiles, and the attributes of users are derived from user profiles. Our approach takes advantage of a variety of user-based characteristics, including:

(i) Number of Followers. This feature defines the number of other users in the network that are following the tweets from your profile. In general, the number of follows determines the attractiveness of a person's profile. Phishers are often less recognized and have a smaller amount of followers than other types of users

(ii) Several Following. This feature determines the set of other user profiles that you are following. When you follow somebody on Twitter, their tweets may appear in your timeline. The Twitter network is aware of who you are following and who is following you

(iii) Age of Account. This feature indicates the date and time at which the account was established

(iv) A follower to Following Ratio. This is the connection between the number of followers and the number of followers for any user profile in a group. The ff ratio is usually lower for normal users, but for frauds, it is greater

$$
\text { FFRatio }=\frac{\text { Number of following }}{\text { a number of followers }}
$$

(v) Reputation. This is the connection between the number of followers and the total number of followers 


$$
\text { Reputation }=\frac{\text { Followers }}{\text { Followers }+ \text { Following }} \text {. }
$$

5.3.2. Content-Based Features. These characteristics are linked to user tweets. Regular users cannot post duplicate material, yet a lot of duplicate tweets are posted by fraudsters. Content-based features are based on stuff written by users. Spam communications may be detected with the content functionality. Fraudsters are malevolent people who distribute a lot of disinformation to members of the network $[17,18]$. The disinformation comprises advertising and harmful links for their goods. Our method uses the different content-based features as follows:

(i) Number of Tweets. A person's total amount of tweets since the first time a profile has been created

(ii) Hashtag Ratio. This is the proportion of tweets with hashtags to the total number of comments submitted and of tweets with one hashtag

$$
\text { Hashtag ratio }=\frac{\text { Duplicate Hashtag }}{\text { Unique Hashtags } \times \text { Tweet count }} .
$$

(iii) URL's Ratio. This corresponds to the number of duplicate URLs in tweets based upon the number of tweets with distinct URLs

$$
\text { Total URLs }=\frac{\text { Hash duplicate URLs }}{\text { Hash unique URLs } \times \text { Tweet count }} .
$$

(iv) Mention Ratio. Users of Twitter account @username are recognized. @username can be tweeted anytime. Fraudsters exploit this function mistakenly to send spam comments to real network members. User communications typically possess a significant number of reply tags that users then believe themselves to be spam users

$$
@ \text { Tweets }=\frac{\text { Tweets containing@ }}{\text { Total number of tweets }} \text {. }
$$

(v) Tweet Frequency. Spammers typically tweet more frequently than legitimate Twitter users, which is a problem

(vi) Spam Words. We employ particular spam phrases and measure the number of times they appear in the tweets of individuals. Fraudsters make use of these spam phrases to convey false information to Internet users
5.4. Analysis Using Artificial Intelligence Model. Once the features and training and test sets have been established, it is essential to select the most appropriate classification approach for the model [19]. Each data set has a perfect classification approach; this would be an exaggeration in the field of analytics; thus, a "fit model" must be created to achieve excellent efficiency based on the data.

5.4.1. Support Vector Machines (SVM). SVM found the approach to data grouping and training and prediction problems as one of the most basic and useful techniques [13]. The input variables are the nearest data point to the judgment area. The most fundamental and significant way to classify the most simple classification models of lower-dimensional transfer learning with discrete classification [10] is the highest range classification. SVM is a simple classification model. Equation (6) used to compute the SVM

$$
y(x)=\operatorname{sign}\left\lfloor\sum_{k=1}^{n} \propto_{k} y_{k} \varphi\left(x, x_{k}\right)+b\right\rfloor,
$$

where $\propto_{k}$ is the positive real constant and $b$ is the real constant.

5.4.2. Artificial Neural Network (ANN). The ANN is a model for computer machinery training based on a biological neural network structure and function. Input and output are changed as the network knowledge flows across the network affects the ANN structure [11]. The ANN is considered a nonlinear data modeling method that models complex input-output relations [14]. Three basic layers are contained in a neural network as shown in Figure 3.

In other words, the corresponding variable $k_{u}(x)$ is given to one-hidden layer MLP

$$
k_{u}(x)=A\left(o_{2}+z_{2}\left(s\left(o_{1}+z_{1 \cdot x}\right)\right)\right) .
$$

" $z_{2}$ " and " $z_{1}$ " are the matrix weight, and " $A$ " is represented by the kernel function, where " $o_{2}$ " and " $o_{1}$ " are the bias objects. Moreover, the hidden state of the $h$ variable is defined as

$$
h(\mathrm{x})=s\left(o+z_{1 . x}\right) .
$$

During this method, iterations are used to ensure the minimum number of potential errors before the necessary input-output mapping has been achieved; a collection of training data, including certain input and associated output vectors, is required here [15]. We learn all model parameters to train an MLP. Let theta $=z_{2}, o_{2}, z_{1}, o_{1}$ is the set of parameters for learning.

5.4.3. Random Forest (RF). The random algorithm of the forest is a managed algorithm for classification. This algorithm generates a forest with many trees, as the name implies [12]. The greater the number of trees in the forests, the same is the case in the random forest classification. The random forest learning algorithm uses the general entity framework aggregation technique. 


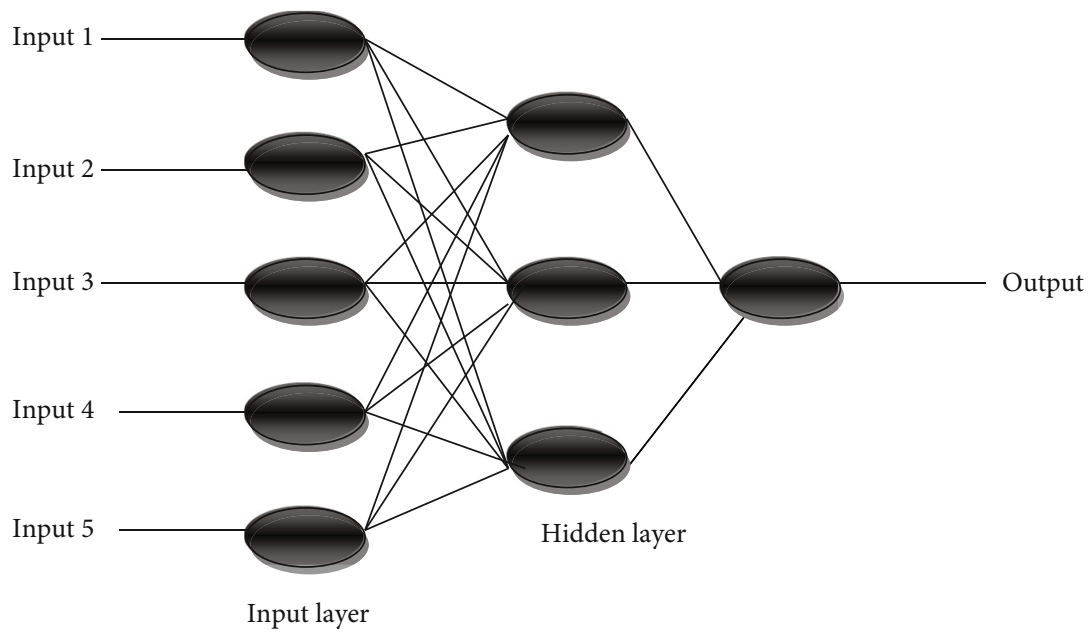

FIGURE 3: Schematic diagram of ANN structure.

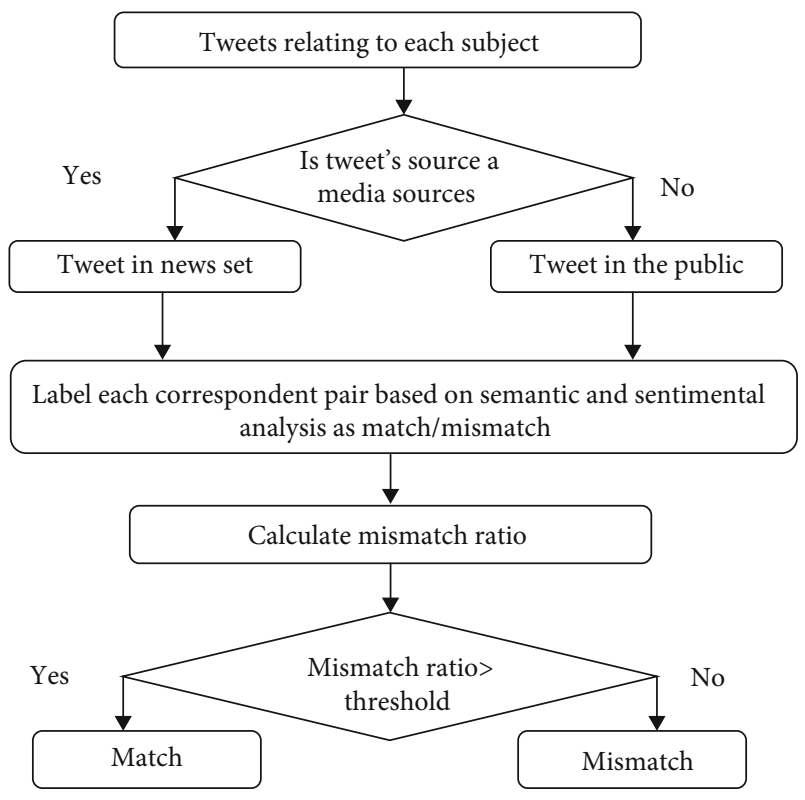

Figure 4: Flow chart of rumor detection in Twitter.

Ensuring a set of $X=x_{1}, \cdots, x_{n}$ with answers $Y=y_{1}, . ., y_{n}$ , repetition of bagging ( $G$ times), the training set substitutes the random sample and suits trees to the samples.

For $g=1, \cdots, G$

(1) Sample, with replacement, $n$ training examples from $X$ and $Y$; call these $X_{g}$ and $Y_{g}$

(2) Train a regression tree $f_{g}$ on $X_{g}$ and $Y_{g}$

Assumptions for unseen samples $x$ may be rendered after training by an averaging of all the specific regression trees by $x^{\prime}$ as shown in Equation (4).

$$
\widehat{f}=\frac{1}{G} \sum_{g=1}^{G} g\left(x^{\prime}\right) .
$$

Or by taking the majority vote in the case of classification trees.

5.5. Evaluation and Assessment. This section describes the authenticity of positive $(\mathrm{P})$ and negative $(\mathrm{N})$. Hacking is described as hit or positive in reality (TP), authorized in reality as negative (TN), and authorized fake websites wrongly as a false positive (PF) or false hit (FP) $[14,15]$.

Accuracy is determined by the classified instances profile ratio over the total profile number as shown in Equation (10)

$$
\text { Accuracy }=\frac{\mathrm{TP}+\mathrm{TN}}{\mathrm{TP}+\mathrm{FN}+\mathrm{FP}+\mathrm{TN}} .
$$

Precision is measured as the proportion of scam profiles accurately estimated against the total number of spam profiles. In other terms, the junk profiles are the proportions that are junk profiles as illustrated in Equation (11)

$$
\text { Precision }=\frac{\mathrm{TP}}{\mathrm{TP}+\mathrm{FP}}
$$

The recall is the percentage of spam profiles accurately estimated against the total amount of real spam profiles as described in Equation (12).

$$
\text { Recall }=\frac{\mathrm{TP}}{\mathrm{TP}+\mathrm{FN}}
$$

F-measure is calculated as the weighted average for both precision and recall as shown in Equation (13).

$$
F-\text { Measure }=\frac{2 \times \text { Precision } \times \text { Recall }}{\text { Precision }+ \text { Recall }} .
$$

ROC Curve Region (AUC) is a well-known classifier consistency assessment indicator. In the case of a random classifier, the AUC value shall equal 0.5, while AUC shall equal 1 for a great classifier as described in Equation (14). 


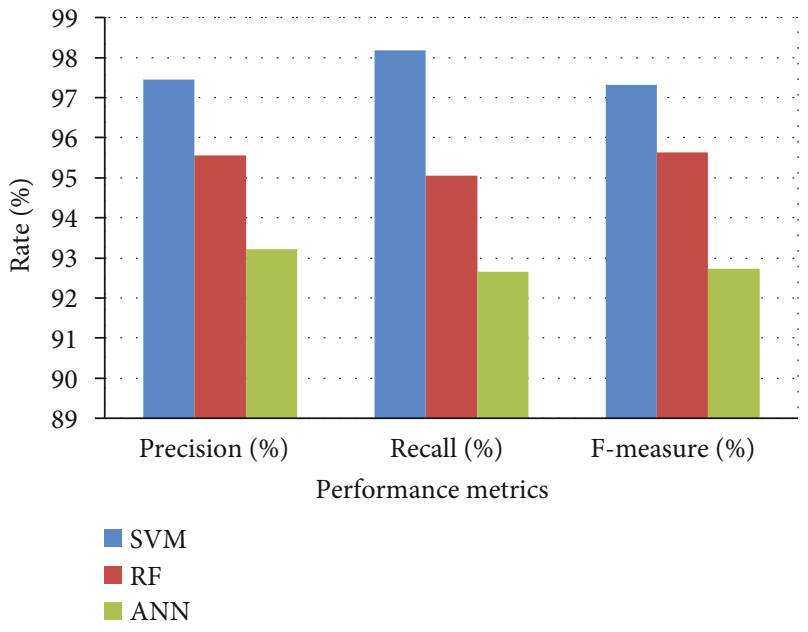

FIGURE 5: Performance of artificial intelligence algorithms using user-based features.

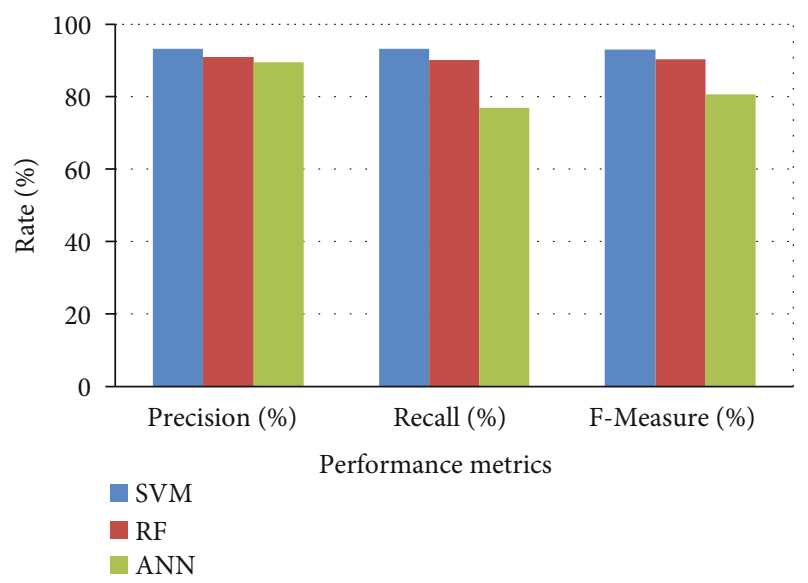

Figure 6: Performance of artificial intelligence algorithms using content-based features.

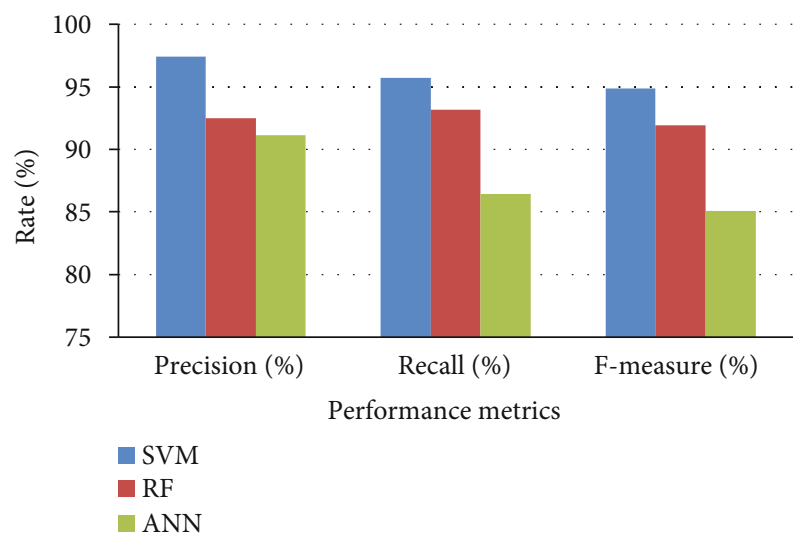

Figure 7: Performance with user-based and content-based features of artificial intelligence algorithms.
TABLE 1: Efficiency of each algorithm utilizing user-based features.

\begin{tabular}{lccc}
\hline Algorithms & Precision (\%) & Recall (\%) & F-measure (\%) \\
\hline SVM & 97.45 & 98.19 & 97.32 \\
RF & 95.56 & 95.06 & 95.64 \\
ANN & 93.21 & 92.65 & 92.73 \\
\hline
\end{tabular}

When comparing the SVM method to the ANN algorithm and the RF algorithm, the SVM algorithm has the highest accuracy, recall, and Fmeasure.

$$
\mathrm{AUC}=\int_{0}^{1} \frac{\mathrm{TP}}{\mathrm{P}} d \frac{\mathrm{FP}}{\mathrm{N}}=\frac{1}{\mathrm{P} \cdot \mathrm{N}} \int_{0}^{1} \mathrm{TP} d \mathrm{FP} .
$$

5.6. Rumor Detection in Twitter. In our context, rumors, where several people think is true, are categorized as any information posted on Twitter, but dispute the news tweets on authenticated news outlets. The present assumption is the basis of our methodology [14]. “Twitter's authenticated TV network accounts would have credible proof compared with the innocent unverified user accounts [20]." The method used by a validated news source for post facts provides a basis for this premise. News agencies verify the material before it is released [7, 9].

They keep the facts they share accountable. They try to uphold their integrity and publish the right information as quickly as possible and take into account that the news affects a broad user base. Twitter verifies their identities and prevents fraud profiles on the news channel. There is also trust in facts from the authenticated media outlet account. Figure 4 gives the flow chart for the algorithm $[13,15]$. The tweets are split into two sets to detect disinformation, both news, and public knowledge, under the principle that their sources are news outlet accounts or otherwise. The news channel's tweets are classified as news tweets, all such tweets are tweeted to the general. Both tweets are linked to semantic and feeling analyses in the news set. Finally, any pair is classified as a fit or mistake of the public cross product as well as new tweet sets.

The difference ratio then measured according to the following formula that represents the extent to which the press and the public differ can be shown in Equation (15).

$$
\text { Mismatch Ratio }=\frac{N}{K}
$$

where $N$ is the amount of polarity public tweets to the contrary and $K$ is the total number of public tweets.

The issue is classified as a match if its mismatch ratio is larger than a threshold value (say 25\%). If a subject is classified as gossip, then the information which conflicts with information from tested sources is believed by the public and is thus published.

\section{Result and Discussion}

The main purpose of this paper is to measure the performance of the spam detection classification models on Twitter. User-based and content-based features are suggested and retrieved from social networking sites to identify spam 
TABLE 2: Efficiency of each algorithm utilizing content-based features.

\begin{tabular}{lccc}
\hline Algorithms & Precision (\%) & Recall (\%) & F-measure (\%) \\
\hline SVM & 93.34 & 93.239 & 93.11 \\
RF & 90.89 & 90.21 & 90.42 \\
ANN & 89.45 & 76.90 & 80.78 \\
\hline
\end{tabular}

When comparing the SVM method to the ANN algorithm and the RF algorithm, the SVM algorithm has the highest accuracy, recall, and Fmeasure.

TABLE 3: Efficiency of each algorithm utilizing user-based features and content-based features.

\begin{tabular}{lccc}
\hline Algorithms & Precision (\%) & Recall (\%) & F-measure (\%) \\
\hline SVM & 97.43 & 95.70 & 94.84 \\
RF & 92.47 & 93.16 & 91.95 \\
ANN & 91.12 & 86.45 & 85.09 \\
\hline
\end{tabular}

When comparing the SVM method to the ANN algorithm and the RF algorithm, the SVM algorithm has the highest accuracy, recall, and Fmeasure.

on Twitter. We analyze classification performance using artificial neural networks, vector support, and random forests. On each algorithm, individual classification trials are conducted. $75 \%$ of the Twitter data sets are picked randomly for training purposes for trials, and the remaining $25 \%$ is selected for classification tests. We utilized a series of measurements termed precision, recall, and F-measure to evaluate the whole methodological procedure.

All categorization algorithms have been developed and validated independently for user-based functions initially. Each classification is then independently trained and assessed for content-based characteristics. All classifications will then be assessed using Figures 5-7 utilizing user-oriented functions and content-based features. Table 1 discusses the user-based performance of the classifier. Table 2 covers the content-based classifier. Table 3 demonstrates user-driven and content-based classifier performance.

\section{Conclusion}

This paper gives a systematic analysis of essential approaches for identifying fraudulent accounts on online social networking sites, such as Facebook (OSNs). In this paper, the primary techniques, as well as a broad range of approaches, that may be used for determining fraudulent accounts in online social networks (OSNs) are addressed. Because of the huge amount of information available on social media platforms, it has become increasingly difficult for consumers to find accurate and useful data in recent years. This paper offers a hybrid collection of spam messages on social networking platforms. We have developed an extensive approach for spam identification in the Twitter dataset to identify spammers. We have employed SVM, ANN, and RF algorithms, as well as hybrid features, such as user-based and content-based features. The recall, precision, and F-measure applying the SVM algorithm are quite effective in our technique. In the future, we plan to broaden our approach to include more types of characteristics and to conduct similar tests on other social media networks that have significant amounts of data.

\section{Data Availability}

The datasets used and/or analyzed during the current study are available from the corresponding author on reasonable request.

\section{Conflicts of Interest}

Conflict of interest is not applicable in this work.

\section{Acknowledgments}

The author thankfully acknowledges the Deanship of Scientific Research, King Khalid University, Abha, Asir, Kingdom of Saudi Arabia, for funding the project under the grant number R.G.P1./74/42.

\section{References}

[1] Y. Boshmaf, D. Logothetis, G. Siganos et al., "Íntegro: leveraging victim prediction for robust fake account detection in large scale osns," Computers \& Security, vol. 61, pp. 142-168, 2016.

[2] A. M. Hemeida, S. Alkhalaf, A. Mady, E. A. Mahmoud, M. E. Hussein, and A. M. Baha Eldin, "Implementation of natureinspired optimization algorithms in some data mining tasks," Ain Shams Eng Journal, vol. 11, no. 2, pp. 309-318, 2020.

[3] N. Kasliwal and T. Bachhav, "Detection of fake accounts of Twitter using SVM and NN algorithms," IEEE Transactions on dependable and secure computing, vol. 5, no. 1, pp. 37-48, 2019.

[4] S. D. P. Reddy, "Fake profile identification using machine learning," International Research Journal of Engineering and Technology (IRJET), vol. 6, no. 12, pp. 1145-1150, 2019.

[5] A. Zubiaga, K. Aker, M. Bontcheva, and R. P. Liakata, "Detection and resolution of rumours in social media: a survey," ACM Computing Surveys (CSUR), vol. 51, no. 2, pp. 1-36, 2018.

[6] P. Kshirsagar and D. S. Akojwar, "Classification and prediction of epilepsy using FFBPNN with PSO," in IEEE International Conference on Communication Networks, Gwalior, 2015.

[7] A. M. Al-Zoubi and H. Faris, "Spam profiles detection on social networks using computational intelligence methods: the effect of the lingual context," in Proceedings of the 19th international conference on World Wide Web, pp. 851-860, Raleigh, NC, April 2010.

[8] H. Faris and Aljarah, "Improving email spam detection using content based feature engineering approach," in Jordan conference on applied electrical engineering and computing technologies (AEECT), pp. 1-6, Aqaba, Jordan, October 2017.

[9] A. el Azab, M. A. Mahmood, and A. el-Aziz, Fraud News Detection for Online Social Networks Web Usage Mining Techniques and Application across Industries, igi global, 2017.

[10] E. Caldeira, G. Brandao, and A. C. M. Pereira, "Fraud analysis and prevention in e-commerce transactions," in Web Congress (LA-WEB), 2014 9th Latin American, pp. 42-49, Minas Gerais, Brazil, 2014. 
[11] P. Kshirsagar, S. Akojwar, and N. D. Bajaj, "A hybridised neural network and optimisation algorithms for prediction and classification of neurological disorders," International Journal of Biomedical Engineering and Technology, vol. 28, no. 4, p. 307, 2018.

[12] P. Kshirsagar and S. Akojwar, "Novel approach for classification and prediction of non linear chaotic databases," in 2016 International Conference on Electrical, Electronics, and Optimization Techniques (ICEEOT), pp. 514-518, Chennai, India, 2016.

[13] P. Kshirsagar and S. Akojwar, "Classification \& detection of neurological disorders using ICA \& AR as feature extractor," International Journal of Scientific Engineering and Science (IJSES), vol. 1, no. 1, 2015.

[14] J. Jiang, C. Wilson, X. Wang et al., "Understanding latent interactions in online social networks," in Proceedings of the 10th ACM SIGCOMM Conference on Internet Measurement, ACM, pp. 369-382, Melbourne, Australia, 2019.

[15] M. Praveena, R. Asha Deepika, and C. Sai Raghavendhar, "Analysis on prediction of heart disease using data mining techniques," Journal of Advanced Research in Dynamical and Control Systems, vol. 10, no. 2, pp. 126-136, 2018.

[16] A. Kundu, S. Panigrahi, S. Sural, and A. K. Majumdar, "Blastssaha hybridization for credit card fraud detection," IEEE Transactions on Dependable and Secure Computing, vol. 6, no. 4, pp. 309-315, 2018.

[17] C. Buntain and J. Golbeck, "Automatically identifying fake news in popular twitter threads," in Proceedings of the IEEE International Conference on Smart Cloud, pp. 208-215, New York, 2017.

[18] S. Tschiatschek, A. Singla, M. Gomez Rodriguez, A. Merchant, and A. Krause, "Fake news detection in social networks via crowd signals," in Proceedings of the World Wide Web Conferences, pp. 517-524, France, 2018.

[19] A. Munther, "A preliminary performance evaluation of Kmeans, KNN and EM unsupervised machine learning methods for network flow classification," International Journal of Electrical and Computer Engineering (IJECE), vol. 6, no. 2, p. 778, 2016.

[20] S. Abu-Nimeh, T. Chen, and O. Alzubi, "Malicious and spam posts in online social networks," Computer, vol. 44, no. 9, pp. 23-28, 2011. 